\author{
María Inés Nin Márquez* \\ ORCID: 0000-0003-2635-8169 \\ Buenos Aires, Argentina \\ Carina Rossa** \\ ORCID: 0000-0001-7290-0217 \\ Vaticano
}

\title{
Educazione interpersonale
}

\section{Interpersonal Education}

Summary: Recent contributions from neuroscience and their implications on school teaching have encouraged openness to new knowledge, to the need for new skills and to the revamping of teaching, placing educational relationship at the centre of everything. The brain has been designed to learn through sharing experiences. The purpose of these pages is hence to see if there is a correlation between mental development and relationality. In that sense, as Chiara Lubich's pedagogy highlights, educational goal is to develop personal identity starting from the encounter with the other. Besides, interpersonal relationships and emotions will help to improve learn-

* Prof. María Inés Nin Márquez PhD, medical doctor specialized in Nutrition, Post-Rational cognitive Therapist, Counselor, Professor at UAI (Universidad Abierta Interamericana, Buenos Aires). Indirizzo: Ciudad de Buenos Aires AR, Av. San Juan 951, C1147 AAH; e-mail: inesnin25@gmail.com.

** Carina Rossa PhD, laureata in Psicopedagogia alla Universidad Catolica Argentina, Dottore in Psicopatologia dello sviluppo e trasformazione sociale alla Libera Università Maria Santissima Assunta (LUMSA) a Roma, Coordinatrice Fondazione Pontificia Scholas Occurrentes. Indirizzo: Città del Vaticano. Piazza San Calisto 16, Città del Vaticano, e-mail: carina. rossa@scholasoccurrentes.org. 
ing. Neuroscience and cognitive neuroscience have demonstrated there is an interconnection between perception, emotion and cognition during school learning. Therefore, we think that valuation of relational and emotional dimensions in education can improve the processes of teaching and learning. In fact, in the last decades, studies and research about human brain function and teaching and learning processes have led to new findings that nowadays are modifying current pedagogy. The progress on science says that interpersonal skills development can improve children's brain development. The relationship with others and other attachment figures is the enabler to allow or inhibit the organisation of neuronal connections, the capability to activate them as a response to stimulus and allow the expression of brain structures genetically determined. This scenario presents the development of prosocial behaviour, which optimise and postulate the capacity for empathy, promoted by Chiara Lubich as behavioural categories of the Art of Love.

Keywords: education; interpersonal neurobiology; brain plasticity; mirror neurons; prosocial behaviours.

Sommario: I recenti contributi delle neuroscienze e le loro implicazioni per la didattica hanno motivato l'apertura a nuovi saperi, la necessità di nuove competenze e il bisogno di rinnovamento della didattica stessa, mettendo al centro di tutto la relazione educativa. Il cervello è stato disegnato per apprendere attraverso le esperienze di condivisione. Lo scopo di queste pagine è quello di verificare l'esistenza di una correlazione tra lo sviluppo mentale e la relazionalitá. In questa direzione, l'approccio educativo di Chiara Lubich sottolinea che l'obiettivo dell'educazione è quello di sviluppare l'identità personale a partire dall'incontro con l'altro. A suo giudizio, inoltre, sia le relazioni interpersonali che le emozioni aiuteranno ad imparare meglio. Le neuroscienze e le scienze cognitive hanno dimostrato l'interconnessione che esiste tra percezione, emozione e cognizione nell'apprendimento scolastico. $\mathrm{Si}$ pensa perciò che la valorizzazione delle dimensioni relazionali ed emotive nella didattica possa migliorare i processi d'insegnamento e d'apprendimento. In effetti, negli ultimi decenni, gli studi e le ricerche sul funzionamento del cervello umano e sui processi d'apprendimento hanno condotto a nuove scoperte che stanno modificando l'educazione attuale. I progressi delle scienze affermano che lo sviluppo delle capacità interpersonali può migliorare lo sviluppo del cervello dei bambini. È il rapporto con gli altri - e con quanti si fanno carico dei bambini - che promuove o inibisce l'organizzazione dei circuiti neurali, la capacità di attivarli in risposta agli stimoli, e che permette l'espressione delle strutture cerebrali determinate geneticamente. In questo scenario si presenta lo sviluppo di comportamenti prosociali, che ottimizzano 
e postulano la capacità di empatia, promossi da Chiara Lubich come categorie comportamentali dell'Arte di Amare.

Parole chiave: educazione; neurobiologia interpersonale; plasticità neuronale; neuroni specchio; prosocialità.

Chiara Lubich, insegnante italiana ${ }^{1}$ e fondatrice del Movimento dei Focolari $^{2}$ presente in varie aree culturali, ha dedicato tutta la sua vita alla formazione delle giovani generazioni. Secondo Lubich l'educazione deve puntare a quella che lei chiama la "finalità educativa", la "meta più alta": "Che tutti siano uno"; essa viene considerata "la finalità delle finalità" perché raggiungerla significa "diventare, come individui e come comunità, capaci di collaborazione, di incontro, di dialogo, di servizio"'. Da ciò emerge la fondamentale importanza che nella sua visione riveste la dimensione relazionale nell'ambito educativo. Il suo approccio potrebbe, infatti, essere definito come una educazione interpersonale, sempre aperta alla più ampia reciprocità sociale e globale, che trova il suo cuore nella relazione.

La formazione dell'identità relazionale richiede la convergenza di due componenti: autonomia e apertura all'altro da me; lo sviluppo delle proprie potenzialità identitarie e la capacità di dialogare con l'altro diverso da me. Questo processo di formazione della persona è forse il principale scopo dell'educazione a tutti i livelli. La finalità dell'educazione consiste, afferma Lubich, nel poter formare l'uomo in modo che egli salvaguardi la sua autonomia, ma allo stesso tempo sappia essere un uomo-relazione ${ }^{4}$. E ancora, in occasione della laurea honoris causa in psicologia, afferma che per un indi-

${ }^{1}$ Cf. Franco de Battaglia, “La Maestra Silvia negli anni di scuola 1939-1943”, in: Essere educatori. Coraggio di una presenza, ed. Michele De Beni (Roma: Citta Nuova, 2013), 17-27.

2 Cf. Armando Torno, Portarti il mondo tra le braccia. Vita di Chiara Lubich (Roma: Citta Nuova, 2012).

${ }^{3}$ Chiara Lubich, "Il Carisma dell'Unità e la Pedagogia", in: Chiara Lubich, La Dottrina spirituale (Roma: Città Nuova, 2006), 318.

${ }^{4}$ Chiara Lubich, "El carisma de la unidad y la pedagogía. Washington, 10 de noviembre de 2000" (Fragmento de la lección realizada el 10 de noviembre de 2000 en la Universidad Católica de América, de Washington, con motivo de la concesión del doctorado honoris causa en Pedagogía), http://centrochiaralubich.org/es/documentos/textos/147-scritto-es/2039-el-carisma-de-la-unidad-y-la-pedagogia.html [access: 12.10.2018]. 
viduo non è possibile acquisire la sua propria identitá se non ha attorno a sé altri che lo riconoscano come soggetto ${ }^{5}$.

Può sorgere una domanda: queste sono solo affermazioni di un' autorevole esponente della spiritualità contemporanea, che poi non hanno alcun riscontro scientifico e quindi sono lontane dalla realtá? Oppure sono idee scientificamente fondate in particolare in ambito neuroscientifico e psicologico?

In queste pagine cercheremo di mettere in luce se esiste una tale correlazione e, in modo specifico, se esiste una relazione tra lo sviluppo mentale, l'apprendimento e la relazionalità.

\section{Il cervello come organo sociale}

Il cervello umano è un organo sociale. Così come gli esseri umani esistono in relazione con gli altri, anche il cervello esiste in relazione con altri cervelli ${ }^{6}$. In quanto esseri umani, ci siamo evoluti per esistere come esseri sociali. I processi mentali sono un prodotto delle nostre connessioni neurali interne e della nostra comunicazione interpersonale in connessione con gli altri ${ }^{7}$.

Taylor indica la caratteristica della natura umana nel suo carattere marcatamente dialogico. Il processo da cui si genera l'identità personale non può essere mai in termini 'monologici'. Difatti il linguaggio come condizione umana, per essere tale, ha bisogno del continuo interscambio con 1'altro ${ }^{8}$. A questo riguardo S. A. Mitchell afferma: "La mente è composta da configurazioni relazionali, si può comprendere l'individuo solo sullo sfondo delle sue relazioni passate e presenti"".

Se i singoli neuroni non si relazionano con gli altri neuroni, appassiscono, muoiono e si tagliano fuori. Allo stesso modo, un simile processo si

${ }^{5}$ Chiara Lubich, "Lezione per la laurea honoris causa in Lettere (psicología), Malta, 26 febbraio 1999”, Nuova Umanità 122 (1999): 186.

${ }^{6}$ Louis Cozolino, Susan Sprokay, "Neuroscience and Adult Learning", New Directions for Adult and Continuing Education 2 (2006): 11-19.

7 Daniel J. Siegel, The developing mind: Toward a neurobiology of interpersonal experience (New York: Guilford, 1999).

${ }^{8}$ Charles Taylor, "La politica del riconoscimento", in: Jürgen Habermas, Charles Taylor, Multiculturalismo. Lotte per il riconoscimento (Milano: Feltrinelli, 1998), 9-62.

9 Stephen A. Mitchell, Gli orientamenti relazionali in psicoanalisi (Torino: Bollati Boringhieri, 1993), 5. 
verifica negli esseri umani. Se non ci connettiamo o non abbiamo relazioni con gli altri, iniziamo ad "atrofizzarci"". La comunicazione modella direttamente il cervello di un bambino che si sviluppa. Secondo Siegel, "la mente sorge a partire dai processi neurofisiologici che hanno luogo nella sostanza cerebrale e dai processi che emergono dalle relazioni interpersonali". Per gli educatori, questi concetti si rivelano essenziali giacché possono aiutare lo sviluppo integrale dei bambini ${ }^{11}$.

Le esperienze relazionali hanno un'influenza molto rilevante sul cervello. Infatti i circuiti responsabili della percezione sociale sono collegati a quelli che controllano la creazione di significato. L'esperienza interpersonale ha un ruolo organizzativo nel determinare lo sviluppo della struttura cerebrale nelle prime fasi della vita e della funzione cerebrale per tutta la vita ${ }^{12}$. L'esperienza attiva i neuroni e modella la struttura del cervello, che cambia continuamente durante la vita. Essa, inoltre, attiva i geni che sintetizzano le proteine in grado di modellare la struttura del cervello ${ }^{13}$.

\section{Plasticità cerebrale}

Recenti scoperte delle neuroscienze suggeriscono che il cervello è plastico, cioè aperto a continue influenze provenienti dall'ambiente ${ }^{14}$. Questa plasticità può comportare non solo la creazione di nuove connessioni sinaptiche tra neuroni, ma anche la crescita di nuovi neuroni nel corso della vita ${ }^{15}$.

Alcuni studi rivelano come possono aver luogo i cambiamenti del cervello, come l'esperienza mentale (immagine) possa alterare la struttura fisica del cervello (connessioni sinaptiche). Questa neuroplasticità fa sì che la con-

${ }^{10}$ Daniel J. Siegel, "Toward an interpersonal neurobiology of the developing mind: attachment relationships, "mindsight," and neural integration, UCLA School of Medicine", Infant Mental Health Journal 1-2 (2001): 67-94.

11 Siegel, The developing.

12 Ibidem.

${ }^{13}$ Eric R. Kandel, “A New Intellectual Framework for Psychiatry”, American Journal of Psychiatry 4 (1998): 457-469; Charles A. Nelson, Floyd E. Bloom, "Child Development and Neuroscience", Child Development 5 (1997): 970-987; Siegel, “Toward an interpersonal”.

${ }^{14}$ Helen Barbas, "Anatomic basis of cognitive-emotional interactions in the primate prefrontal cortex", Neuroscience and Biobehavioral Reviews 19 (1995): 499-510; Francine M. Benes, "Human brain growth spans decades", American Journal of Psychiatry 155 (1998): 1489.

15 Siegel, "Toward an interpersonal". 
sapevolezza interna, prodotta da un'immagine autogenerata, possa alterare l'attività delle connessioni neurali in specifiche regioni del cervello ${ }^{16}$.

La sintonizzazione tra l'insegnante e l'allievo riesce a creare un cambiamento nel cervello, rendendolo capace di incorporare nuove informazioni. Tale evidenza è stata dimostrata in vari studi dai quali è emerso come la cura e il supporto di un insegnante, insieme alla sua capacità di valorizzare l'allievo, sono capaci di causare significative trasformazioni cerebrali ${ }^{17}$. Lo sviluppo del cervello diventa così un processo dinamico, trovandosi in costante riorganizzazione durante tutto il ciclo della vita ${ }^{18}$.

\section{Neurobiologia interpersonale}

La neurobiologia interpersonale è un approccio che cerca di unire gli aspetti neurobiologici, l'esperienza soggettiva e le relazioni umane. Daniel Siegel parla di neurobiologia interpersonale in base alla sua teoria della mente. A suo giudizio la mente emerge dal cervello nella misura in cui questo è modellato e cambiato dai rapporti interpersonali. Infatti Daniel Siegel sottolinea che "la mente sorge a partire dal flusso di informazioni ed energia all'interno dello stesso cervello e tra due o più cervelli" 19 .

Le interazioni emotive con figure di attaccamento sono molto importanti nella prima infanzia giacché aiutano a sviluppare il senso di sé. La mente si sviluppa nella interazione con un altro significativo, e gli stati mentali degli altri vengono rappresentati all'interno del cervello nel funzionamento neuronale ${ }^{20}$. Da questa interazione con 1'“oggetto", si creano nel cervello del bambino delle mappe neurali di sé stesso. Queste mappe neurali sono l'essenza di come il cervello crea immagini della propria mente o di altre menti (attitudini, credenze, pensieri,ecc.), in un processo rappresentativo chiamato

16 Siegel, The developing.

${ }^{17}$ Cozolino, Sprokay, "Neuroscience".

${ }^{18}$ Beatriz Rodríguez Vega, Alberto Fernández Liria, Carmen Bayón Pérez, "Neurobiología interpersonal", in: Manual de Psiquiatría, eds. Tomás Palomo, Miguel Ángel Jiménez-Arriero (Madrid: Ene Life Publicidad), 3-8.

19 Daniel J. Siegel, “An Interpersonal Neurobiology Approach to Psychotherapy”, Psychiatric Annals 36 (2006): 4.

${ }^{20}$ Valerie Stone, Simon Baron-Cohen, Robert T. Knight, "Frontal lobe contributions to theory of mind", Journal of Cognitive Neuroscience 10 (1998): 640-656. 
"mindsight"21. Questa capacità complessa si sviluppa durante l'infanzia e può arricchirsi durante tutta la vita ${ }^{22}$. Un esempio di ciò possiamo trovarlo nella relazione tra madre e bambino quando, nelle interazioni reciproche, il bambino sorride e la madre sorride in un interscambio tra i due. In questa esperienza si hanno delle emozioni crescenti che stimolano i processi metabolici nel cervello, consentendo una maggiore crescita e sviluppo, non solo nel cervello, ma nel corpo intero. Succede diversamente nei bambini che sono stati abbandonati e trascurati. Infatti il loro sviluppo cerebrale e la crescita del corpo diminuiscono ${ }^{23}$. Situazioni di stress, che si creano a scuola o nell'ambiente d'apprendimento, oppure i ricordi negativi di esperienze passate, possono indebolire o bloccare l'apprendimento dell'allievo. Invece, quando l'insegnante ha un atteggiamento di cura e di incoraggiamento, si crea un favorevole stato biologico, dovuto alla secrezione di neuropeptidi nel cervello dell'allievo, che lo rendono capace di apprendere e di incorporare nuova informazione.

\section{Teoria della mente}

Da quanto fin qui esposto emerge che, nelle relazioni umane, la mente ed il cervello sono uniti tra di loro da flussi di energia che ricevono e danno informazione. Possiamo dire con Siegel che la mente "viene creata nell'interazione tra il neurofisiologico e l'interpersonale quando i cervelli interagiscono tra loro" 24 .

Alcuni studi neuroscientifici ci aiutano ad intendere quanto le relazioni interpersonali influiscano sullo sviluppo del cervello. Sarebbe pure di estremo interesse conoscere i meccanismi per i quali il funzionamento del cervello influisce sulla intersoggettività. La Teoria della Mente, proposta da Premack e Woodruff nel $1978^{25}$, spiega questa capacità cognitiva degli individui di attribuire stati mentali agli altri e a se stessi. Questo termine si riferisce all'abilità di comprendere e prevedere il comportamento degli altri, le loro

${ }^{21}$ Kenneth Aitken, Colwyn Trevarthen, "Self-other organization in human psychological development", Development and Psychopathology 9 (1997): 653-678.

22 Siegel, "Toward an interpersonal".

${ }^{23}$ Ruth Buczynski, Louis Cozolino, "The Neuroscience of Psychotherapy" (2011), www. nicabm.com (A Teleseminar Session with Louis Cozolino, PhD and Ruth Buczynski, PhD).

${ }^{24}$ Siegel, "An Interpersonal", 4.

${ }^{25}$ David Premack, Gui Woodruff, "Does the Chimpanzee have a Theory of Mind?", Brain and Behavioral Sciences 1 (1978): 515-526. 
emozioni ed intenzioni. La teoria della mente favorisce pure la comprensione dell'inganno e delle menzogne e, a sua volta, aiuta l'individuo a mentire e ad ingannare ${ }^{26}$. Infatti, noi, esseri umani siamo dotati di una capacità innata che ci permette di essere in relazione. Possiamo comunicare trasmettendo i nostri desideri e, dall'altra, possiamo capire le credenze ed intenzioni degli altri ${ }^{27}$.

Nell'evoluzione della teoria della mente si registra un' importante svolta grazie alla ricerca fatta da Baron-Cohen, Leslie e Frith ${ }^{28}$. Questi autori hanno scoperto che le persone con autismo non hanno una ToM (Theory of mind) a causa della loro incapacità di attribuire stati mentali a se stessi e agli altri, non sono in grado di prevedere e spiegare i comportamenti. Questa ipotesi si è basata in parte su Alan Leslie, autore di un'analisi delle abilità cognitive nei bambini normali, finalizzata a comprendere il gioco della finzione. Per Leslie e Frith, dal primo anno di vita i bambini sviluppano la capacità di elaborare rappresentazioni sulle rappresentazioni (meta-rappresentazioni) degli eventi del mondo reale ${ }^{29}$.

Il fatto che i bambini da 12 a 24 mesi comprendano l'azione intenzionale costituisce un dato già dimostrato da vari studi pubblicati negli ultimi decenni. I bambini, dopo i 14 mesi, possono offrire spontaneamente il loro aiuto a una persona che cerca di completare un compito, ma non riescono nel loro intento ${ }^{30}$. Dai 12 mesi in poi, invece, sono in grado di aiutare uno sperimentatore 'ignorante' ${ }^{31}$. Queste ricerche fanno vedere che c'è già un'intenzionalità condivisa nei bambini di questa etá e per alcuni autori questa costituisce l'origine della ToM nella nostra specie ${ }^{32}$.

${ }^{26}$ Santiago Uribe Ortiz, Mónica Gómez Botero, Olber Eduardo Arango Tobón, “Teoría de la mente: una revisión acerca del desarrollo del concepto", Revista Colombiana de Ciencias Sociales 1 (2010): 28-37.

27 Vittorio Gallese, "Neuroscienze e fenomenologia", http://old.unipr.it/arpa/mirror/pubs/ pdffiles/Gallese,Neuroscienze_e_fenomenologia_finale.pdf [access: 14.04.2018].

${ }^{28}$ Simon Baron-Cohen, Alan Leslie, Uta Frith, "Does the autistic child have a 'theory of mind'?", Cognition 21 (1985): 37-46.

29 Alan Leslie, Uta Frith, “Autistic children's understanding of seeing, knowing and believing”, British Journal of Developmental Psychology 6 (1988): 315-324.

${ }^{30}$ Felix Warneken, Michael Tomasello, “Altruistic Helping in Human Infants and Young Chimpanzees", Science 31 (2006): 1301-1303.

${ }^{31}$ Ulf Liszkowski, Malinda Carpenter, Michael Tomasello, "Twelve-Month-Old communicate Helpfully and Appropriately for Knowledgeable and Ignorant Partners”, Cognition 108 (2008): 732-739.

${ }^{32}$ Michael Tomasello, The Cultural Origins of Human Cognition (Cambridge, Mass.: Harvard University Press, 1999; Michael Tomasello, Why We Cooperate (Massachusetts: MIT Press., 2009). 
La Teoria della Mente quindi è un elemento chiave per comprendere la comunicazione interpersonale nei processi di insegnamento-apprendimento. Nelle relazioni interpersonali interpretiamo continuamente il comportamento dell'altro, assumendo i suoi stati mentali, come idee, credenze, desideri, intenzioni, interessi. Interpretiamo il comportamento di qualcuno come risultato di certi pensieri che ha, dei suoi sentimenti o desideri ${ }^{33}$. Questo sistema di conoscenza permette di dedurre desideri e sentimenti e di interpretarli.

Da quello che abbiamo detto finora, si capisce che la promozione della ToM nella scuola ha dei vantaggi per l'apprendimento scolastico. Bruner ${ }^{34}$ ha proposto che una buona ToM aiuta i bambini ad imparare a riflettere sui propri processi di pensiero e apprendimento. Ciò significa che otterrebbero delle capacità metacognitive che aiutano l'apprendimento. Più tardi è stato dimostrato che negli anni prescolari esiste una relazione positiva tra il livello della ToM e le abilità metacognitive nella scuola elementare ${ }^{35}$. Questa abilità umana quindi si dimostra fondamentale sia per l'apprendimento culturale che per la relazione interpersonale. Nell'insegnamento si cerca di trasmettere qualcosa e per questo l'insegnante e l'allievo devono intendere la mente l'uno dell'altro, inferire le proprie conoscenze ed interessi e viceversa ${ }^{36}$.

\section{Neuroni - specchio}

Un'altra scoperta fondamentale, che sostiene questa teoria, è stata effettuata a Parma nel 1995 da un'équipe di neurobiologi italiani, guidati da Rizzolatti nel corso di una ricerca con scimmie addestrate per afferrare oggetti. Con un microelettrodo impiantato nella corteccia premotoria (dove si piani-

33 Angel Rivière, Objetos con mente (Madrid: Alianza, 1991); Angel Rivière, Maria Núñez, La mirada mental (Buenos Aires: Aique, 1997); Andrew Whiten, Natural Theories of Mind (Oxford: Blackwell, 1991); Juan Carlos Gómez, El desarrollo de la mente en los simios, los monos y los niños (Madrid: Morata, 2007); Emilio García García, Mente y cerebro (Madrid: Síntesis, 2001); Emillio García García, et al., Nuevas perspectivas científicas y filosóficas sobre el ser Humano (Madrid: Universidad Comillas, 2007).

34 Jerome Bruner, The Culture of Education (Cambridge, Mass.: Harvard University Press, 1996).

35 Wolfgang Schneider, "The Development of Metacognitive Knowledge in Children and Adolescents: Major Trends and Implications for Education", Mind Brain and Education 2 (2008): 114.

${ }^{36}$ Emilio García García, "Neuropsicologia y educación. De las neuronas espejo a la teoría de la mente", Rev. de psicologia y educación 3 (2008): 69-90. 
ficano e si avviano i movimenti), hanno registrato l'attività elettrica di alcuni neuroni. In un'occasione il dispositivo di registrazione si attivò senza che la scimmia svolgesse nessuna attività. Gli bastava vedere un'altra scimmia che stava facendo un' azione simile. Gli scienziati avevano scoperto un tipo di neuroni sconosciuti fino ad allora, chiamati neuroni speculari. Questi neuroni reagiscono quando l'azione e il suo obiettivo sono visti insieme e non si attivano quando gli oggetti si afferrano senza scopo. Questo vuol dire che le cellule rappresentano l'obiettivo unito al movimento ${ }^{37}$.

I neuroni specchio vengono attivati nel processo pedagogico. Il cervello di un bambino, che vede un'azione da parte del suo insegnante, simula mentalmente in forma sincronica le azioni presentate dal suo insegnante e al contempo le interpreta. Grazie alla sua neurofisiologia, il bambino deduce le intenzioni dell'insegnante e così assimila i concetti e i processi con cui interagisce a livello cerebrale e sociale.

Alcuni studi correlano empatia e simpatia col funzionamento di questa particolare zona del cervello che ospita il sistema dei neuroni specchio. Gli stessi autori della scoperta autorizzano a considerare che i neuroni specchio sono alla base dell'intersoggettività e dell'empatia ${ }^{38}$. In un certo senso, "la simulazione incarnata può essere considerata come il correlato funzionale dell'empatia" ${ }^{\text {"39 }}$. Invece, per Baron-Cohen si devono prendere in considerazione anche altri fattori che stanno alla base del circuito dell'empatía e che non sono solo di natura organica. Il circuito dell'empatia, per esempio, può essere compromesso da alcune esperienze accadute nei primi anni di vita nei quali si è avuto poco sostegno affettivo, o nei casi di diverse forme di violenza o di abuso. Tuttavia, benché ridotta da eventi traumatici, la capacità empatica può migliorare ogni volta che si cerca di capire i pensieri e i sentimenti degli altri.

Basandosi sulla risonanza magnetica funzionale (FMRI), questo autore sottolinea che il livello di empatia dipende dal "Circuito Empatico", le cui aree celebrali sarebbero danneggiate nei soggetti borderline e in quelli psicopatici. In questi soggetti si avrebbe un' incapacità di comprendere le conseguenze delle proprie azioni. Quindi l'empatia va oltre il rispecchiamento automatico, costituendo la possibilità di comprendere gli stati mentali degli

${ }^{37}$ Ibidem.

38 Vittorio Gallese, "Dai neuroni specchio alla consonanza intenzionale. Meccanismi neurofisiologici dell'intersoggettività", Rivista di Psicoanalisi 1(2007): 197-208; Giacomo Rizzolatti, "Neuroni specchio ed empatia", intervento del 22 settembre 2012 a "Pordenone legge", http://www.youtube.com/watch?v=02szjHTch98 [access: 19.06.2018].

39 Gallese, "Dai neuroni", 197-208. 
altri attraverso l'attivazione di uno specifico circuito neuronale dell'empatia $^{40}$. I meccanismi di simulazione ci permettono, come strumento privilegiato, di poter condividere a livello esperienziale gli stati mentali altrui. Una serie di nuovi studi sembrano confermare l'ipotesi che nell'autismo infantile vari di questi meccanismi appaiono alterati.

Tornando ai neuroni specchio, essi sono capaci di attivarsi anche quando l'osservazione non è totalmente visibile. Per esempio, se non si vede l'interazione tra la mano, il braccio o il piede e l'oggetto, essa può essere "inferita" dal soggetto che osserva ${ }^{41}$. Solo che in questo caso non si parla di un'inferenza logica, ma del risultato di una simulazione motoria. L'attivazione del programma motorio di chi sta osservando corrisponde ad un' azione che non si può vedere nella sua totalità. Ma che, vista solo parzialmente, ne consente la comprensione. Una serie di studi hanno dimostrato che un meccanismo analogo di rispecchiamento è presente anche nel cervello umano, e si basa su una specifica corrispondenza, punto per punto, di un'area del corpo con un'area specifica del sistema nervoso centrale. Quando osserviamo azioni eseguite da altri con la bocca, la mano, o il piede, attiviamo corrispondenti regioni del nostro sistema motorio ${ }^{42}$.

Grazie ai meccanismi cruciali di rispecchiamento e simulazione siamo arrivati alla comprensione dell'intersoggettività, dove l'altro è vissuto come un "altro sé". Assegniamo implicitamente all'altro lo status di un'altra soggettività che condivide con noi la condizione umana e quindi possiamo rapportaci in piena reciprocità. Infatti, questi meccanismi neuronali non si attivano soltanto nel dominio dell'azione, ma anche in quello delle emozioni o delle sensazioni. L'intersoggettività si trasforma così nel fondamento ontologico della vita ${ }^{43}$.

\section{Dall'empatia alla prosocialità, dalla prosocialità all'arte di amare di Chiara Lubich}

L'empatia è una disposizione cognitiva ed emotiva che facilita la qualità dei rapporti interpersonali. Per Martin L. Hoffman il fatto di condividere

40 Simon Baron-Cohen, La scienza del male. L'empatia e le origini della crudeltá (Milano: Raffaelo Cortina, 2012).

${ }^{41}$ Maria Alessandra Umiltà et al., “'I know what you are doing': a neurophysiological study”, Neuron 32 (2001): 91-101; Warneken, Tomasello, “Altruistic Helping”, 1301-1303.

${ }^{42}$ Gallese, "Dai neuroni”, 197-208.

${ }^{43}$ Ibidem. 
l'angoscia di chi patisce - compassione - è quello che provoca l'azione di aiuto $^{44}$. Così appare il rapporto tra empatia e prosocialità. Per comportamenti prosociali si intende:

Quei comportamenti che, senza la ricerca di ricompense esterne, estrinseche o materiali, favoriscono altre persone o gruppi, secondo i criteri di questi, o mete sociali obiettivamente positive e che aumentano la probabilità di generare una reciprocità positiva di qualità e solidale nelle relazioni interpersonali o sociali conseguenti, salvaguardando l'identità, la creatività e l'iniziativa degli individui o gruppi implicati ${ }^{45}$.

Il rapporto tra empatia e prosocialità è bidirezionale. L'empatia facilita la ricorrenza degli atti prosociali ed allo stesso tempo si può affermare che le persone che agiscono prosocialmente impareranno progressivamente a ottimizzare la propria capacità empatica.

Il grado di empatia secondo Hoffman è collegato ai giudizi morali e cioè agli atteggiamenti etici delle persone. La comprensione cognitiva del pensiero dell'altro o la sperimentazione di sentimenti simili possono promuovere il comportamento di aiuto verso l'altro e questa disposizione va motivata ulteriormente secondo i principi e norme che costituiscono l'insieme di valori che indirizzano i comportamenti delle persone.

Nel pensiero e negli scritti di Lubich si trova una categoria, "l'arte di amare" 46 , che ha affinità con l'empatia e la prosocialità; si tratta della "diplomazia della carità" o il "Farsi Uno" che Lubich descrive così:

Farsi uno con il prossimo in quel completo oblio di sé che possiede - senza avvedersene e senza curarsene - colui che ricorda l'altro, il prossimo. Questa è la "diplomazia della carità", che ha della diplomazia ordinaria molte espressioni e manifestazioni, per cui dice non tutto quello che potrebbe dire, perché al fratello non piacerebbe e non sarebbe gradito; sa attendere, sa parlare, arrivare allo scopo [...] Ha essa però un timbro essenziale e caratteristico che la differenzia da quella di cui parla il mondo, per il quale dir diplomazia spesso è sinonimo

${ }^{44}$ Martin L. Hoffman, "Development of prosocial motivation: Empathy and guilt", in: The development of prosocial behavior, ed. Nancy Eisenberg (New York: Academic Press, 1982), 282-313.

${ }^{45}$ Robert Roche Olivar, Psicologia y Educacion para la Prosocialidad (Buenos Aires: Ed. Ciudad Nueva, 1996), 16.

${ }^{46}$ Cf. Chiara Lubich, Arte di amare (Roma: Citta Nuova, 2005). 
di reticente o addirittura falso. La diplomazia divina ha questo di grande e di suo, forse di solo suo: che è mossa dal bene dell'altro ed è priva quindi d'ogni ombra di egoismo ${ }^{47}$.

L'arte di Amare - comportamenti che si orientano al bene dell'altro privi di egoismo che per la loro capacità empatica provocano una reciprocità positiva - somiglia nella sua definizione ai comportamenti prosociali. L'allenamento di essi possono essere la base di un'educazione interpersonale perché conducono al dialogo, alla collaborazione e all'unità salvaguardando la propria identità.

\section{Riconoscimento}

Da quanto fin qui detto e da quanto sostenuto dalla ricerca neuroscientifica, emerge la fondamentale importanza, nell'atto educativo, di considerare la relazione con l'altro come uno spazio all'interno del quale nasce la mente umana e si fonda la nostra identità. L'altro deve essere riconosciuto per nascere, svilupparsi ed arrivare alla realizzazione di sé che porta al benessere ${ }^{48}$.

Ciò che rende possibile la relazione umana e lo sviluppo dell'identità personale, è il poter riconoscere l'altro differente da me, nella sua specifica dignità, dandogli fiducia, in una tensione empatica capace di rinunciare al proprio punto di vista e ai propri interessi ${ }^{49}$.

Il mutuo riconoscimento, che avviene in una relazione d'amore, consentendo il costituirsi della piena soggettività e di un'identità sempre nuova, si realizza con l'apprezzamento ed il rispetto. Invece, una relazione caratterizzata dalla noncuranza e dall'imposizione della propria volontà con prepotenza, comporta l'annullamento e l'incapacità di andare oltre se stessi ${ }^{50}$.

${ }^{47}$ Chiara Lubich, La dottrina spirituale (Roma: Città Nuova, 2006), 331.

48 Piero A. Cavaleri, Vivere con l'altro per una cultura della relazione (Roma: Città Nuova, 2007); Cozolino, Sprokay, "Neuroscience".

${ }^{49}$ Cavaleri, Vivere; Pierpaolo Donati, "Il dono in famiglia e nelle altre sfere sociali", in: Dono e perdono nelle relazioni familiari e sociali studi interdisciplinari sulla famiglia, eds. Eugenia Scabini, Giovanna Rossi (Milano: Vita e pensiero, 2000), 55-132.

${ }^{50}$ Enrico Molinari, Piero A. Cavaleri, Il dono nel tempo della crisi. Per una psicologia del riconoscimento (Milano: Raffaello Cortina, 2015), 32-33; Paola Gaetano, "Dialettiche della reciprocità. Lo stile affettivo nelle organizzazioni di significato personale", 2011, in XIII convegno di psicologia e psicopatologia post-razionalista "all you need is love": la rilevanza 
Per questo motivo l'educazione al "difficile", come compito che coinvolge sia l'educando che l'educatore, è un altro punto fondamentale della pedagogia di Chiara Lubich $^{51}$.

Anche in campo educativo - in tanti modi - con forme di iperprotettività, si tende a preservare i minori da qualsiasi difficoltà, abituandoli a vedere la vita come una strada in discesa, facile, comoda. In realtà, li si lascia in forte disagio di fronte alle inevitabili prove della vita e, in particolare, li si rende passivi e renitenti rispetto alle responsabilità che ogni essere umano deve assumersi di fronte a se stesso, al prossimo, alla società. L'educazione al difficile: un impegno che coinvolge sia l'educando che l'educatore ${ }^{52}$.

In particolare, educare alla capacità di gestire i conflitti consente di modificare un'esperienza negativa e frustrante in un'esperienza che, se trasformata, diventa un'occasione di maturazione personale e di scoperta dell'altro ${ }^{53}$.

Ciò accade quando il ' $\mathrm{Tu}$ ' si sente riconosciuto e riesce a ricambiare questo dono con l'apprezzamento e il mutuo rispetto. In questo modo l'Altro potrà sviluppare la sua vera identità e la sua libertà ${ }^{54}$.

In conclusione è possibile sottolineare come le affermazioni di Chiara Lubich siano fondate e trovino riscontro in diverse ricerche scientifiche tendenti a dimostrare come il rapporto di reciproco riconoscimento promuova l'organizzazione dei circuiti neurali, aumentando la capacità cerebrale e quindi l'apprendimento.

\section{Bibliografia}

Aitken, Kenneth, Colwyn Trevarthen. "Self-other organization in human psychological development". Development and Psychopathology 9 (1997): 653-678. Barbas, Helen. "Anatomic basis of cognitive-emotional interactions in the primate prefrontal cortex". Neuroscience and Biobehavioral Reviews 19 (1995): 499-510 .

dello stile affettivo in psicoterapia post-razionalista (Atti del convegno a cura di Mario Antonio Reda e Luca Canestri).

${ }^{51}$ Lubich, "El carisma".

52 Ibidem.

${ }^{53}$ Cavaleri, Vivere.

${ }^{54}$ Gaetano, "Dialettiche". 
Baron-Cohen, Simon, Alan Leslie, Uta Frith. "Does the autistic child have a 'theory of mind'?”. Cognition 21 (1985): 37-46.

Baron-Cohen, Simon. La scienza del male. L'empatia e le origini della crudeltà. Milano: Raffaelo Cortina, 2012.

Battaglia, Franco de. "La Maestra Silvia negli anni di scuola 1939-1943". In: Essere educatori. Coraggio di una presenza, ed. Michele De Beni, 17-27. Roma: Citta Nuova, 2013.

Benes, Francine M. "Human brain growth spans decades". American Journal of Psychiatry 155 (1998): 1489.

Bruner, Jerome. The Culture of Education. Cambridge, Mass.: Harvard University Press, 1996.

Buczynski, Ruth, Louis Cozolino. "The Neuroscience of Psychotherapy" (2011). www.nicabm.com.

Cavaleri, Piero A. Vivere con l'altro per una cultura della relazione. Roma: Città Nuova, 2007.

Cozolino, Louis, Susan Sprokay. "Neuroscience and Adult Learning". New Directions for Adult and Continuing Education 2 (2006): 11-19.

Donati, Pierpaolo. "Il dono in famiglia e nelle altre sfere sociali". In: Dono e perdono nelle relazioni familiari e sociali studi interdisciplinari sulla famiglia, eds. Eugenia Scabini, Giovanna Rossi, 55-132. Milano: Vita e pensiero, 2000.

Gallese, Vittorio. "Dai neuroni specchio alla consonanza intenzionale. Meccanismi neurofisiologici dell'intersoggettività". Rivista di Psicoanalisi 1 (2007): 197_ -208 .

Gallese, Vittorio. "Neuroscienze e fenomenologia". http://old.unipr.it/arpa/mirror/ pubs/pdffiles/Gallese,Neuroscienze_e_fenomenologia_finale.pdf [access: 14.04. 2018].

García García, Emilio. Mente y cerebro. Madrid: Síntesis, 2001.

García García, Emillio, et al. Nuevas perspectivas cientificas y filosóficas sobre el ser Humano. Madrid: Universidad Comillas, 2007.

García García, Emilio. "Neuropsicologia y educación. De las neuronas espejo a la teoría de la mente". Rev. de psicologia y educación 3 (2008): 69-90.

Gómez, Juan Carlos. El desarrollo de la mente en los simios, los monos y los niños. Madrid: Morata, 2007.

Hoffman, Martin L. "Development of prosocial motivation: Empathy and guilt". In: The development of prosocial behavior, ed. Nancy Eisenberg, 282-313. New York: Academic Press, 1982.

Kandel, Eric R. "A New Intellectual Framework for Psychiatry". American Journal of Psychiatry 4 (1998): 457-469. 
Leslie, Alan, Uta Frith. “Autistic children's understanding of seeing, knowing and believing”. British Journal of Developmental Psychology 6 (1988): 315-324.

Liszkowski, Ulf, Malinda Carpenter, Michael Tomasello. "Twelve-Month-Old communicate Helpfully and Appropriately for Knowledgeable and Ignorant Partners". Cognition 108 (2008): 732-739.

Lubich, Chiara. Arte di amare. Roma: Citta Nuova, 2005.

Lubich, Chiara. "Lezione per la laurea honoris causa in Lettere (psicología), Malta, 26 febbraio 1999”. Nuova Umanità 122 (1999): 177-189.

Lubich, Chiara. "El carisma de la unidad y la pedagogía. Washington, 10 de noviembre de 2000". http://centrochiaralubich.org/es/documentos/textos/147-scrittoes/2039-el-carisma-de-la-unidad-y-la-pedagogia.html [access: 4.04.2018].

Lubich, Chiara. "Il Carisma dell'Unità e la Pedagogia". In: Chiara Lubich, La Dottrina spirituale, 265-270. Roma: Città Nuova, 2006.

Lubich, Chiara. La dottrina spirituale. Roma: Città Nuova, 2006.

Mitchell, Stephen A. Gli orientamenti relazionali in psicoanalisi. Torino: Bollati Boringhieri, 1993.

Premack, David, Guy Woodruff. "Does the Chimpanzee have a Theory of Mind?". Brain and Behavioral Sciences 1 (1978): 515-526.

Molinari, Enrico, Piero A. Cavaleri. Il dono nel tempo della crisi. Per una psicologia del riconoscimento. Milano: Raffaello Cortina, 2015.

Nelson, Charles A., Floyd E. Bloom. "Child Development and Neuroscience". Child Development 5 (1997): 970-987.

Rivière, Angel. Objetos con mente. Madrid: Alianza, 1991.

Rivière, Angel, Maria Núñez. La mirada mental. Buenos Aires: Aique, 1997.

Rizzolatti, Giacomo. "Neuroni specchio ed empatia", intervento del 22 settembre 2012

a "Pordenone legge". http://www.youtube.com/watch?v=02szjHTch98 [access: 19.06.2018].

Roche Olivar, Robert. Psicologia y Educacion para la Prosocialidad. Buenos Aires: Ed. Ciudad Nueva, 1996.

Rodríguez Vega, Beatriz, Alberto Fernández Liria, Carmen Bayón Pérez. "Neurobiología interpersonal”. In: Manual de Psiquiatría, eds. Tomás Palomo, Miguel Ángel Jiménez-Arriero, 107-113. Madrid: Ene Life Publicidad, 2009.

Siegel, Daniel J. The developing mind: Toward a neurobiology of interpersonal experience. New York: Guilford, 1999.

Siegel, Daniel J. "Toward an interpersonal neurobiology of the developing mind: attachment relationships, "mindsight," and neural integration, UCLA School of Medicine". Infant Mental Health Journal 1-2 (2001): 67-94.

Siegel, Daniel J. “An Interpersonal Neurobiology Approach to Psychotherapy”. Psychiatric Annals 36 (2006): 1-18. 
Schneider, Wolfgang. "The Development of Metacognitive Knowledge in Children and Adolescents: Major Trends and Implications for Education". Mind Brain and Education 2 (2008): 114-121.

Stone, Valerie, Simon Baron-Cohen, Robert T. Knight. "Frontal lobe contributions to theory of mind". Journal of Cognitive Neuroscience 10 (1998): 640-656.

Taylor, Charles. "La politica del riconoscimento". In: Jürgen Habermas, Charles Taylor, Multiculturalismo. Lotte per il riconoscimento, 9-62. Milano: Feltrinelli, 1998.

Tomasello, Michael. The Cultural Origins of Human Cognition. Cambridge, Mass.: Harvard University Press, 1999.

Tomasello, Michael. Why We Cooperate. Massachusetts: MIT Press., 2009.

Torno, Armando. Portarti il mondo tra le braccia. Vita di Chiara Lubich. Roma: Citta Nuova, 2012.

Uribe Ortiz, Santiago, Mónica Gómez Botero, Olber Eduardo Arango Tobón. “Teoría de la mente: una revisión acerca del desarrollo del concepto". Revista Colombiana de Ciencias Sociales 1 (2010): 28-37.

Umiltà, Maria Alessandra, et al.. “'I know what you are doing': a neurophysiological study”. Neuron 32 (2001): 91-101.

Warneken, Felix, Michael Tomasello. "Altruistic Helping in Human Infants and Young Chimpanzees”. Science 31 (2006): 1301-1303.

Whiten, Andrew. Natural Theories of Mind. Oxford: Blackwell, 1991. 
